\title{
Los Zapatos del Zapatero: Los Sueños del Analista con el Paciente ${ }^{1}$
}

\author{
Mariia Demianchuk ${ }^{2}$ \\ Viena, Austria
}

\begin{abstract}
Este trabajo examina el fenómeno de soñar con el paciente tanto desde un nivel teórico como práctico. Comienza con la introducción de la cuestión del "uso de los sueños" en el arte, la cultura y la literatura. La segunda parte esboza las aportaciones teóricas de Ogden, Gabbard, Bion, Zwiebel y otros respecto a la cuestión de los sueños y la contratransferencia. La relación especial de Freud con Rome e Italia es abordada. La tercera parte presenta una viñeta clínica en la cual, tras presentar a la paciente, se analizarán dos sueños con la paciente. El trabajo concluye con los comentarios finales.
\end{abstract}

Palabras clave: Soñar con el paciente, análisis de los sueños, contratransferencia, histeria, escisión.

This paper examines the phenomena of dreaming about the patient on both theoretical and practical levels. It begins by introduction to the topic 'use of the dreams' in art, culture and literature. The section two traces the theoretical inputs of Ogden, Gabbard, Bion, Zwiebel and others around the topic of dreaming and countertransference. Special relationship of Freud to Rome and Italy is discussed. The section three provides a clinical vignette, where after introducing the patient, two dreams about the patient are analysed. The paper is topped off with the concluding remarks.

Key Words: Dreaming about the patient, dream analysis, countertransference, hysteria, splitting.

English Title: The shoes for the shoemaker: on analyst's dreams about the patient

\section{Cita bibliográfica / Reference citation:}

Demianchuk, Mariia. (2016). Los zapatos del Zapatero: Los sueños del Analista con el Paciente. Clínica e Investigación Relacional, 10 (3): 770-779. [ISSN 1988-2939] [Recuperado de www.ceir.info ] DOI: 10.21110/19882939.2016.100312

1 Leído en la Conferencia de IARPP en Roma, June 12, 2016: THE ARTS OF TIME. RELATIONAL PSYCHOANALYSIS AND FORMS OF VITALITY IN CLINICAL PROCESS. Traducción castellana del original inglés a cargo de Andrea Iturriaga Medrano.

2 Dirección de contacto: contact@psychotherapeutin.work 


\section{Introducción}

¿Fue Chuang Tzu quién soñó que era una mariposa o fue la mariposa que soñó ser Chuang Tzu?

Todas las personas sueñan. Sigmund Freud consideró su libro "La Interpretación de los Sueños" (1900) como su trabajo más importante, ya que destapa la entrada a la vida inconsciente de las personas. Este libro fue el más revisado de todos sus trabajos. Freud postuló que los sueños son la vía regia al inconsciente del paciente. Comúnmente se piensa que a través de los sueños uno puede alcanzar importantes insights, especialmente en los dominios del arte, la literatura y la ciencia. Pensar por un momento en las películas de David Lynch o en la tabla periódica de Mendeleev. En la "Interpretación de los Sueños" argumenta que la condición fundamental de la creación poética comprende una actitud muy similar" a la libre asociación que esperamos en análisis y que se manifiesta en los sueños. Los sueños pertenecen al despacho donde tiene lugar el análisis. En otro trabajo (Demianchuk, 2015) discuto acerca de la variedad de interpretaciones psicoanalíticas acerca de los sueños así como las implicaciones clínicas que conllevan.

\section{Revisión Teórica}

Ogden (2004a: 858) sugirió que convertirse en analista conlleva un proceso de "soñar uno mismo más profundamente acerca de la existencia". Se sostenía que tanto analista como paciente necesitaban "consultar con la almohada" la sesión (Ogden \& Gabbard, 2009: 313). En la tradición de la teoría Bioniana (1962), se propone emplear el término "soñar" para referirse a la forma más arcaica de pensamiento. ¿Qué pasa entonces con los sueños del analista que aparecen fuera de la terapia? Este trabajo se propone a mirar de cerca el fenómeno de "soñar con el paciente". Si aquellos sueños del paciente que incluyen al analista se atribuyen como parte de la transferencia y reciben dicha atribución en la teoría psicoanalítica del desarrollo temprano, los sueños del analista con el paciente han supuesto un tabú durante mucho tiempo, de igual manera que lo fue la cuestión de la contratransferencia en psicoanálisis hasta 1950 cuando el polémico trabajo de Paula Heimann (y el trabajo de Heinrich Racker) la reconoció como una parte importante de la relación y del proceso terapéutico.

En el desarrollo de la teoría psicoanalítica han tenido lugar cambios de paradigmas. Uno de ellos es el cambio de una persona, un sujeto, a sujeto-sujeto, interpersonal, el discurso relacional. Tras varias décadas, con el rol ya aceptado y creciente de la contratransferencia, es momento de considerar también los sueños del analista con el paciente. ¿Deben ser 
desestimados como efectos irrelevantes de la terapia? ¿Podemos hacer uso de ellos? Si así es, ¿qué tipo de uso? ¿Cómo descifrarlos y utilizarlos en la terapia? Algunos analistas como Ralf Zwiebel (2002) los entiende como "los restos de la neurosis del psicoanalista", siendo "perturbaciones" de la dinámica transferencia-contratransferencia. En cambio en la teoría relacional, la situación psicoterapéutica es co-construida por el psicoanalista y el paciente. Al igual que el paciente, el analista también tiene niveles de funcionamiento consciente e inconsciente. En este trabajo me centro en los sueños del analista como el espacio (de juego) para dos "inconscientes", el de analista y paciente. Los sueños son una fuente de información muy significativa acerca de lo que ocurre en la terapia y, sino más importante, de aquello que no ocurre en la terapia, aquello que es reprimido, escindido, oculto, pero que encuentra su vía de manifestación en forma de sueño. Este trabajo, de alguna manera, es la puesta en escena de la puesta en escena del inconsciente de mi paciente en mi sueño, como parte de mi propia inconsciente.

Existe una relación especial entre Italia y el Padre del Psicoanálisis: la temática de Roma es una de las más interesantes en "La Interpretación de los Sueños" (Gay, 1989: 132). Freud sueña con la celebración de un congreso en Roma junto con Fliess, "para escuchar acerca de las leyes de la existencia primero desde la Ciudad Eterna". Su anhelo por Roma lo denominó como "profundamente neurótico" (Ibíd. 133). Freud introdujo la analogía entre la historia de Pompeya (su sepultura y la posterior excavación) y los procesos psíquicos que Freud descubrió - sepultura por medio de la represión y la excavación con el análisis. Sugirió que "un sueño puede ser interpolado en la concatenación psíquica que puede ser retoralimentada desde una idea patológica en las evocaciones de un paciente". El siguiente paso era tratar el propio sueño como un síntoma, y aplicarle el método de la interpretación que se hubiera elaborado para dicho síntoma" (Freud 1997: 14, 15) En cambio, yo veo el sueño como un lugar, la escena para un síntoma. Freud abordó la posible complicación que pudiera surgir del análisis de los sueños propios y la relación con detalles manifiestos de la propia vida psíquica para las interpretaciones de un extraño.

Clínicamente, encuentro mucho apoyo en la sugerencia de Bollas acerca de que el paciente histérico usa la psique del analista para hacerle sentir la experiencia temprana, la experiencia perturbadora, la cual es desalojada esperando que se vuelva a recibir de vuelta pero mejor organizada. El paciente traslada al analista su "self desgraciado", es el objeto interno el que tiene que ser tratado más plenamente, Esta reacción contratransferencial (a la que pertenecen los sueños) es la conversión histérica, la cual tiene que ser devuelta por el psicoanalista (Bollas, 1987). 


\section{Viñeta Clínica}

A fin de ilustrar la universalidad de la interpretación de los sueños también para una persona "más o menos normal" (así es como Freud se llamó a sí mismo (1987:18)), llevó a cabo el "auto-análisis" del famoso sueño de Irma. Aunque en el sueño de Freud Irma es una figura que condensada numerosos significados, también era su ex-paciente. Al analizar mis propios sueños tomé éste como ejemplo. En este punto quisiera aportar cierta información básica acerca de mi paciente, los sueños acerca de ella son el material que presento para esta viñeta clínica.

Amber, la paciente, tiene 27 años y es una chica irlandesa que vive en Viena; ha estado realizando su Master en Traducción durante los últimos cuatro años en la Universidad de Viena. Vive con su novio Allan, un cocinero austriaco, ambos trabajan juntos en un restaurante. La paciente es la segunda de tres hermanos, tiene un hermano mayor y una hermana menor, María. Cuando Amber tenía siete años sus padres se divorciaron. Había sentido a su madre como una mujer fría, convencida de que sus hijos le habían arruinado su figura, nunca estuvo para ellos. Ella y sus hermanos habían convivido con cuidadoras mejicanas, una de ellas se llamaba María también. No conserva recuerdos de la infancia con su padre. En su anamnesis, además de un temprano diagnóstico de depresión, había tenido anorexia, atracones y conductas autolesivas. Su intención consciente de una terapia era centrarse en tres aspectos importantes: la baja autoestima, los problemas de ira y la insatisfacción ante sus propias decisiones. Tuve dos supervisores para este caso, siendo este mi caso final para conseguir mi licencia como psicoanalista. La paciente estuvo en terapia psicoanalítica conmigo durante dos años y medio, con una regularidad de dos veces en semana y en diván. A nivel diagnóstico encuentro en ella una "personalidad histeriforme" (Hojer-Pedersen, 1965) con aspectos narcisistas o "la llamada histérica buena con una estructura depresiva" (Zetzel, 1964). Las características principales de su psicodinámica son la agresión oral, la duda respecto al género, su identificación con el padre castrado y la rivalidad entre los hermanos.

Tuve el primer sueño en la tercera fase de la terapia cuando estaba experimentando dificultades con su diagnóstico.

El sueño:

Es una mañana tranquila en el sur de Italia, vivo en el segundo piso con mi novio, él también está ahí. Tenemos una invitada, una reportera, Emma A. Estamos sentados en la cocina. Escucho a alguien tocar la puerta, bajo y saludo afectuosamente a un joven guapo. En el sueño 
sé que es ella, mi paciente. Es la primera vez que está en nuestra casa así que se la enseño. También le muestro el dormitorio, es grande y luminoso, las sábanas están aún revueltas tras la noche. Observo cómo ella reacciona. La paciente parece ruborizarse. Vamos a la cocina a desayunar, nosotros, tres mujeres, sentadas alrededor de la mesa y mi novio fregando los platos. Emma A. habla acerca de los derechos de las mujeres. Así es como acaba el sueño.

Freud sugiere analizar los sueños por partes, esto trataré de hacer a continuación. Trataré de separar aquello que pertenece a mis asociaciones personales y aquello que puede tener conexiones con el proceso psicoterapéutico de la paciente en particular.

Es una mañana tranquila en el sur de Italia, vivo en el segundo piso con mi novio, él también está ahí. Italia, y especialmente Roma, era siempre mi destino soñado. Lo asocio a bellas mujeres femeninas, una cultura rica y comida exquisita. El segundo piso puede representar a mis padres, o hermanos mayores, la posición-cerca-al-Panteón. En ocasiones me han confundido con ser italiana. Al igual que la paciente, la familia de su madre proviene de Italia y las madres italianas tienen mucho poder. El ambiente de la escena es parecido al de "la Belleza Robada" de Bertolucci, en la cual la chica busca a su padre desconocido tras morir su madre. Bertolucci estuvo durante varios años en tratamiento analítico.

Tenemos una invitada, una reportera, Emma A. Estamos sentados en la cocina. Esto es una alusión a la paciente histérica de Freud - Emmy von R. Emma es 5-7 años más joven que yo (como la paciente). La conocí al comentar acerca de unos temas en su programa de TV, aquí aparece conexión con otro síntoma histérico: la dramatización (Inszenierung (En alemán)), en la cual estoy implicada. Sorprendentemente, los dos temas sobre los que fui entrevistada por Emma fueron el amor como enfermedad y el problema de la dependencia maligna, ambos aspectos muy importantes en la histeria. Emma A. es reconocida en Ucrania como reportera feminista. Es una de las pocas periodistas que en Ucrania habla acerca de la comunidad LGTB. Por tanto, ella habla de aquellos temas escindidos y reprimidos dentro de la sociedad patriarcal, tal y como ocurre en la dinámica histérica. Creo que en el sueño ella representa la bisexualidad y los trabajos clásicos de Freud: Estudios sobre la Histeria y Tres Ensayos sobre la Teoría Sexual. Aunque el número "tres" pertenece a la sintaxis de la histeria, la triangulación Edípica y somos tres en la cocina.

Escucho a alguien tocar la puerta, bajo y saludo afectuosamente a un joven guapo. El chico es rubio, igual que la paciente; tiene entre 14-16 años, se mueve tímidamente, es alto y delgado. Sé que siente la inseguridad propia de la adolescencia y también una baja autoestima. Al ser consciente del hecho de su fragilidad, me acerco a él con una aceptación incondicional materna. 
En el sueño sé que es ella, mi paciente. Es la primera vez que está en nuestra casa así que se la enseño. También le muestro el dormitorio, es grande y luminoso, las sábanas están aún revueltas tras la noche. Observo cómo ella reacciona. La paciente parece ruborizarse. Hay un evidente sobrepaso de los límites del encuadre analítico, en el sueño ella no es mi paciente, pero yo sé que es mi paciente. Mi deseo en este momento es el de darle a conocer la sexualidad genital, pero su ruborización ${ }^{1}$ es el indicativo de que su sexualidad se despierta por un momento y vuelve a reprimirse. Muy a menudo, en la contratransferencia la siento como alguien muy frágil, a veces quiero abrazarla, llevarla a mi casa y cuidar de ella, como una madre. En una ocasión, durante una sesión me dijo que si yo fuera madre sería muy "comprensiva, entregada y divertida." Por ello, supongo que este sueño cumple dos deseos: el suyo- tener una mejor madre, el mío- ser una mejor madre para ella. Pero este cambio de género merece una mayor discusión. Las dudas respecto al género aparecen en su paleta de síntomas. La desigualdad de género era una cuestión que aparecía muy a menudo en nuestras sesiones, ella creía que los hombres tienen muchas más oportunidades en la vida, o al menos, en la cultura Occidental. En referencia a la psicodinámica familiar ella siempre había querido ser un chico como su hermano mayor, el cual era el preferido de su madre o un hombre como su padre desaparecido (en el discurso melancólico- ser el objeto que ha sido perdido evitando así el duelo) o diferenciarse como alguien más fuerte que su hermana pequeña, rivalidad que está enormemente presente en su relación con otras mujeres y en la propia terapia. Su edad en el sueño es la edad con la que empezaron sus cuestiones orales: anorexia y bulimia acompañadas de conductas autolesivas. Podríamos decir que se acerca mucho a la personalidad borderline, pero recordemos a Bollas (2000), quién sugirió que la histeria sólo desaparecía para volver en forma de borderline. La manera masculina de atravesar la adolescencia rara vez toma este camino. En el sueño cumplo otro de sus deseos - ser un hombre. ¿Era realmente mío el deseo de que ella fuera un hombre? No lo creo, la transferencia - contratransferencia erótica es complicada de manejar. Pero puede que los hijos sean más fáciles de amar para una madre, la rivalidad es alejada con los impulsos eróticos. Ella responde a la cuestión lacaniana de la histeria: soy hombre o mujer, en esta ocasión en el sueño en congruencia a su cuerpo de joven adolescente. Ante la habitación "parental" ella se ruboriza. Si reformulamos el famoso dicho de Freud acerca de la histeria, lo podríamos leer como: "Donde la excitación debería estar - hay repugnancia y vergüenza" (Freud 1995: 184).

Vamos a la cocina a desayunar, nosotros, tres mujeres, sentadas alrededor de la mesa y mi novio fregando los platos. Emma A. habla acerca de los derechos de las mujeres. Esta escena indica la estructura familiar de la madre, la hermana y la paciente y el "débil" y acallado padre. La cocina, como escena de este sueño, es un indicativo de la cuestión de la oralidad. 
El segundo sueño tuvo lugar en la cuarta fase de la terapia, cuando yo trataba de abordar la transferencia lateral en la terapia:

Sé que llego tarde a la sesión. Mi despacho se encuentra en la planta alta del edificio (en la séptima u octava). Es una habitación más bien pequeña con una gran ventana y sillas como las del colegio. He dado cita a dos de mis pacientes a la misma hora, el martes a las $2 \mathrm{pm}$. En la habitación hay dos de mis pacientes mujeres: una de ellas es Amber, mi paciente, la otra... no pude acordarme de su nombre hasta después de despertarme. Pero en el sueño sé dónde puedo encontrar su nombre, es Sharon Stone. Se están divirtiendo sin mí: corren alrededor de las mesas, ríen, casi les estoy molestando. Me siento culpable. Me disculpo por mi error. Les pregunto quién de las dos puede esperar una hora, Sharon Stone responde que ella puede. Le pido entonces que salga, pero me ignoran y siguen corriendo como dos adolescentes. Amber me enseña el trabajo del Coloquio Il acerca de su caso, sus reflexiones, escrito en francés y con tinta. Me está diciendo: "Léelo si puedes". No puedo ya que no hablo francés y esto me frustra incluso más. Sé que en algún lugar de la misma planta está Felix de Mendelssohn (mi supervisor varón) escribiendo su libro. Voy a buscarle y le cuento la situación. Me escucha y después de dice: "¿Quién quieres que sea yo en esta situación?" Te estás ridiculizando a ti misma, tratando de convertirme en el buen padre. ¡Eres capaz de resolver la situación por ti misma!" No me doy por vencida y permanezco ahí junto a su puerta. Finalmente él accede y me acompaña hasta mi despacho, me siento aliviada. Entramos en la habitación y el sueño acaba.

Sé que llego tarde a la sesión. Mi despacho se encuentra en la planta alta del edificio (en la séptima u octava). Los padres de mi paciente se divorciaron cuando ella tenía 7 años. Nuestra clínica, en la que llevamos a cabo la terapia, está en la octava planta del edificio.

Es una habitación más bien pequeña con una gran ventana y sillas como las del colegio. La habitación me recuerda a la clase en la que daba inglés y alemán en el instituto, los idiomas que ambas hablamos.

He dado cita a dos de mis pacientes a la misma hora, el martes a las 2pm. En la habitación hay dos de mis pacientes mujeres: una de ellas es Amber, mi paciente, la otra... no pude acordarme de su nombre hasta después de despertarme. Pero en el sueño sé dónde puedo encontrar su nombre, es Sharon Stone. Yo sabía dónde podía buscar el nombre (en mi pared, dónde también solían estar colgados mis esquemas conceptuales para mi tesis). Mi primera asociación con respecto a ella es un dicho que creía erróneamente que era de ella: "Nos convertimos en los hombres con los que queríamos casarnos en nuestra juventud". Sharon Stone es también muy sexy y femenina, famosa por su papel en "Instinto Básico", película en la que enseña sus genitales. Mi paciente se debate en su feminidad, tiene un aspecto 
andrógino aunque atractivo, nunca lleva sujetador, hace boxeo y se pregunta acerca de qué encuentran los hombres atractivo en las mujeres. Este tema de lo que atrae a los hombres aparecía muy a menudo en nuestras sesiones.

Se están divirtiendo sin mí: corren alrededor de las mesas, ríen, casi les estoy molestando. La paciente tiene una hermana menor, cuando ellas eran pequeñas solían estar solas. Yo también tengo una hermana menor de la edad de mi paciente, creo que yo tenía una mejor relación con mi hermana, jugábamos mucho juntas. En el sueño juegan a quién es más rápida, más lista, el juego de las diferencias entre hermanos en el cual mi paciente nunca ganaba. Aunque los hermanos siempre permanecen juntos, ellas nunca estuvieron unidas, solían pelearse mucho. En el sueño me siento inútil, creo que también es mi sentimiento contratransferencial cuando nos vemos atrapadas en la dinámica de rivalidad entre hermanas.

Me siento culpable. Me disculpo por mi error. Les pregunto quién de las dos puede esperar una hora, Sharon Stone responde que ella puede. Le pido entonces que salga, pero me ignoran y siguen corriendo como dos adolescentes. Amber me enseña el trabajo del Coloquio II acerca de su caso, sus reflexiones, escrito en francés y con tinta. Me está diciendo: "Léelo si puedes". No puedo ya que no hablo francés y esto me frustra incluso más. La culpa en el analista es a menudo una proyección del paciente histérico. La inutilidad de la madre en esta ocasión, quién no puede controlar a sus hijas, el problema de comunicación en las relaciones madrehija. Ella es mi caso final para el examen, el Coloquio II, para poder obtener mi licencia como psicoanalista. En ese momento yo estaba atravesando dificultades durante la escritura de su caso y por supuesto, un trabajo previamente escrito era algo que me hubiera sido de mucha ayuda. En cambio, ella me lo da escrito en tinta y en francés. Debería pensar en mi supervisora mujer Wolff Bernstein, ella sabe francés y suele escribir con una pluma de tinta. El francés es un idioma que me gustaría aprender, pero por el momento no me ha sido posible. Esto supone ahora rivalidad entre ambas, ella tiene/sabe algo que yo quiero. En la realidad, ella tiene envidia de mi conocimiento de ruso. ¿Entonces por qué en el sueño doy la vuelta a este aspecto aun siendo desfavorable para mí? Debido a la culpa, no exclusivamente de la psicodinámica histérica, sino también de un resto del día: yo falté a una sesión con mi paciente debido a razones objetivas, pero aun así me sentía culpable. Miremos por un momento al juego de nombres: Amber - Stone - Bern - Stein, Amber=Bern (Alemán), Stone=Stein (Alemán) ${ }^{2}$. La disociación forma parte del discurso del histérico, tal y como pronto demostró Charcot (Demianchuk 2016: 9). Aquí nos encontramos con el nombre de mi paciente escindido neuróticamente: femenino Vs. masculino, vida vs. Muerte, poder vs. Dependencia, transferencia vertical vs. Lateral. La escisión queda incorporada en el nombre de mi supervisora, nos da la esperanza de que tras un buen 
psicoanálisis una integración interior es posible (Bernstein es también nombre de una persona).

. Sé que en algún lugar de la misma planta está Felix de Mendelssohn (mi supervisor varón) escribiendo su libro. Voy a buscarle y le cuento la situación. Me escucha y después de dice: "¿Quién quieres que sea yo en esta situación?" Te estás ridiculizando a ti misma, tratando de convertirme en el buen padre. ¡Eres capaz de resolver la situación por ti misma!" No me doy por vencida y permanezco ahí junto a su puerta. Finalmente él accede y me acompaña hasta mi despacho, me siento aliviada. Entramos en la habitación y el sueño acaba. Igual que yo, la figura materna no fue demasiado para pararlas, tuve que traer al padre fálico. La ley del padre según los lacanianos, Nome de Pere, otra conexión con el francés. Considero que este es una de las mayores carencias de mi paciente - la ausencia del padre, no hubo nadie que pudiera tornar su mirada a la madre hasta mirar el nuevo e inmenso mundo, tal y como Jessica Benjamin (1991) diría.

\section{Comentarios Finales}

Tras el análisis de los sueños, traté de aplicar las hipótesis y verbalizarlas en sesión con mi paciente. Por supuesto, esto de ninguna manera es un análisis exhaustivo, pero espero haber mostrado el modo en que podemos pensar los sueños con los pacientes, los cuales pueden servir para obtener una mayor asimilación de los objetos internos del paciente y para un mayor entendimiento de aquello que pertenece a la psicodinámica de la relación terapéutica, pero está escindido por el momento.

Hay un significado peculiar en la interrelación entre sole mender (reparador de suelas) y soul mender (reparador de almas), la profesión del zapatero tiene una connotación semi-sagrada debido a que se les permitía trabajar los domingos igual que a los curas, la razón era que normalmente ellos trabajaban para los curas los domingos. Los inicios del Psicoanálisis comenzaron con el semi-mágico acceso de la hipnosis, pero a día de hoy se trata de una ciencia fascinante en desarrollo.

El psicoanálisis, igual que ocurre con los seres humanos, posee áreas de sensibilidad psíquica, que, también a través de los sueños, pueden ser activadas en psicoterapia. Mi sugerencia es la de tratar esto como material que pertenece al análisis y necesita de interpretación psicoanalítica.

\section{REFERENCIAS}


Benjamin, J. (1991). Father and Daughter: Identification with difference - a contribution to gender heterodoxy. Psychoanalytic Dialogues: The International Journal of Relational Perspectives, 1:3, 277-299.

Bion, W.R. (1962). Learning from experience. In: Seven servants. New York, NY: Aronson.

Bollas, C. (1987). Shadow of the Object. New York: Columbia University Press.

Bollas, C. (2000). Hysteria. London, UK: Routledge.

Demianchuk, M. (2015). Modern Psychoanalytic approaches to dreams and their clinical implications. In W. Owczarski and Z. Ziemann (Ed.), Dreams, Phantasms and Memories (pp. 99-106). Gdansk: Gdansk University Press.

Demianchuk, M. (2016). Hysteria revisited: A Case Study of Orthorexia Nervosa. (Unpublished master thesis). Sigmund Freud University, Vienna.

Etchegoyen, H. (2005). Fundamentals of Psychoanalytic Technique. KARNAC Books., London.

Freud, S. (1997). The Interpretation of Dreams. (A. A. Brill, Trans.). New York, NY: The Macmillan Company. (Original work published in 1900).

Gabbard, G. \& Ogden, T. (2009). On Becoming a Psychoanalyst. International Journal of Psychoanalysis, 90, 311-327.

Gay, P. (ed.) (1995). The Freud reader. London, UK: Vintage.

Ogden, T.H. (2004). This art of psychoanalysis: Dreaming undreamt dreams and interrupted cries. International Journal of Psychoanalysis, 85, 857-78.

Yglesias, R. (1996). Dr. Neruda's Cure for Evil. Boston, MA: Grand Central Publishing.

Zaretsky, E. (2004). Secrets Of The Soul: A social And Cultural History Of Psychoanalysis. New York, NY: Vintage Books.

Zwiebel, R. (2002). Die Träume des Analytikers. In R. Zwiebel und M. Leuzinger-Bohleber (hrsg.), Träume, Spielräume (pp. 110-132). Psychoanalytische Blätter, Bd. 20. Göttingen: Vandenhoeck \& Ruprecht.

Original recibido con fecha: $\quad$ 2-8-2016 Revisado: 5-10-2016 Aceptado: 31-10-2016

NOTAS:

${ }^{1} \mathrm{~N}$. de T: En el texto original la autora utiliza un juego de palabras con el propio nombre de la paciente AMBERassment, en lugar de embarrassment. El juego de palabra nos comunica acerca de la ruborización en cuanto a la sexualidad de Amber, su paciente.

${ }^{2} \mathrm{~N}$. de T: La autora identifica un juego de palabras entre el nombre de su paciente, el apellido de su supervisora y el apellido de Sharon Stone. Amber y Bern (comienzo del apellido de su supervisora) son ambos nombres alemanes para mujer y hombre respectivamente y tienen ciertas similitudes fonéticas y ortográficas. Y por otro lado Stone y Stein (final del apellido de su supervisora) significan piedra en inglés y alemán respectivamente. 